Article

\title{
Sensitivity of the Gravity Model and Orbital Frame for On-board Real-Time Orbit Determination: Operational Results of GPS-12 GPS Receiver
}

\author{
Eunhyouek Kim ${ }^{1, * \mathbb{C}}$, Seungyeop Han ${ }^{1}$ and Amer Mohammad Al Sayegh ${ }^{2}$ \\ 1 Flight Control System Team, Satrec Initiative, Daejeon 305-811, Korea \\ 2 Space Systems Development Department, Mohammed Bin Rashid Space Centre, \\ Dubai 211833, United Arab Emirates \\ * Correspondence: ehkim@satreci.com; Tel.: +82-42-330-6875
}

Received: 26 April 2019; Accepted: 21 June 2019; Published: 28 June 2019

check for updates

\begin{abstract}
This paper describes the sensitivity of both the orbital frame domain selection and the gravity model on the performance of on-board real-time orbit determination. Practical error sources, which affect the navigation solution of spaceborne global positioning system (GPS) receivers, are analyzed first. Then, a reasonable orbital frame (radial, in-track, cross-track (RIC)) is proposed to clearly represent the characteristics of the error in order to improve the performance of the orbit determination (OD) logic. In addition, the sensitivity of the gravity model affecting the orbit determination logic is analyzed by comparison with the precise orbit ephemeris (POE) of the Challenging Minisatellite Payload (CHAMP) satellite, and it is confirmed that the Gravity Recovery And Climate Experiment (GRACE) Gravity Model 03 (GGM03) outperforms the Earth Gravity Model 1996 (EGM96). The effects of both proposed orbit frames and the gravity model on the orbit determination logic are verified using a GPS simulator and observation data from the CHAMP satellite. Moreover, the practical performance of on-board real-time orbit determination logic is verified by updating the software of the spaceborne GPS receiver, GPS-12, on DubaiSat-2 operating at low Earth orbit (LEO). The results show that the position accuracy of on-board real-time orbit determination logic in GPS-12 is improved by $59 \%$, from $12.6 \mathrm{~m}(1 \sigma)$ to $5.1 \mathrm{~m}(1 \sigma)$, after applying the proposed methods. The velocity accuracy is also improved by $57 \%$, from $13.7 \mathrm{~mm} / \mathrm{s}(1 \sigma)$ to $5.9 \mathrm{~mm} / \mathrm{s}(1 \sigma)$.
\end{abstract}

Keywords: DubaiSat-2; CHAMP; LEO; GPS-12 spaceborne GPS receiver; EGM96; GGM03; ECI; RIC; sensitivity; orbit determination

\section{Introduction}

Today, satellites depend entirely on GPS receivers to acquire the current time and to synchronize each subsystem. The GPS Receiver and Processor Package (GPSAC) GPS receiver was installed on Landsat-4 in 1982, and its feasibility has been proved [1,2]. GPS receivers have been applied to various satellites after the first GPS receiver was in operation. To improve the performance of GPS receivers, precise orbit determination (POD) logic has been developed [3]. A GPS receiver was first installed on the Galileo In-Orbit Validation Element-A (GIOVE-A) satellite for the geostation mission in 2006 [4]. The navigation solution generated by the standard positioning service (SPS) logic of the GPS receiver operating at low Earth orbit (LEO) is degraded by ephemeris message error, signal noise, ionospheric time delay, and so on. Sometimes, the spaceborne GPS receiver can experience position errors of several kilometers and velocity errors of several meters per second in the navigation solution, reducing the reliability of the navigation solution [5-7].

The motivation for the spaceborne GPS receiver to have on-board real-time orbit determination logic originates from an emerging requirement to generate a precise navigation solution at the level of 
several meters. The precise navigation solution will be helpful in terms of operating synthetic-aperture radar (SAR) payloads and improving the pointing accuracy. It is possible that there will be a further need to generate a more precise navigation solution in terms of satellite automation. The orbit determination algorithm using an extended Kalman filter with the C/A code pseudo-range measurements of the Topex/Poseidon satellite proved that the position and velocity accuracy can be improved about $20 \mathrm{~m}$ root mean square (RMS) and $0.1 \mathrm{~m} / \mathrm{s}$ RMS, respectively [8]. Gill and Montenbruck used the pseudo-range and carrier phase of L1 as measurement information in the progress of developing the navigation system using extended an Kalman filter and showed that a position accuracy improved by about 25-30 $\mathrm{m}$ for the X-SAT cube satellite [9]. Group and Phase Ionospheric Correction (GRAPHIC) combined a code and carrier measurements to remove the ionospheric time delay, and a position accuracy of about 1 meter was achieved, compared to the 3D precision of $30 \mathrm{~cm}$ for post-processed orbits [10]. These methods, however, are not practical for on-board real-time orbit determination because there is plenty of computation burden and a shortage of memory size. The orbit determination logic, which was developed by Satrec Initiative, was designed to operate in real-time on the GPS-12 spaceborne GPS receiver. Developing on-board real-time orbit determination logic using an extended Kalman filter has improved both the accuracy and stability of the navigation solution [11,12]. Initially, the orbit determination logic tried to remove jerk and noise using the simple J2 orbit model. As a result, position accuracy has been improved by 65\%, from about $70 \mathrm{~m}(1 \sigma)$ to $26 \mathrm{~m}(1 \sigma)$. The kind of orbit perturbation force is summarized in Figure 1, and it is clear that the gravity model takes up the most acceleration at LEO [13]. The high precision orbit propagator (HPOP) orbit model with EGM96 was applied to the orbit determination logic to improve the accuracy of the navigation solution in May 2016. After applying the HPOP on the on-board real-time orbit determination logic of GPS-12, the accuracy of the navigation solution improved by about 50\%, from about $26 \mathrm{~m}(1 \sigma)$ to about $13 \mathrm{~m}(1 \sigma)$ [12].

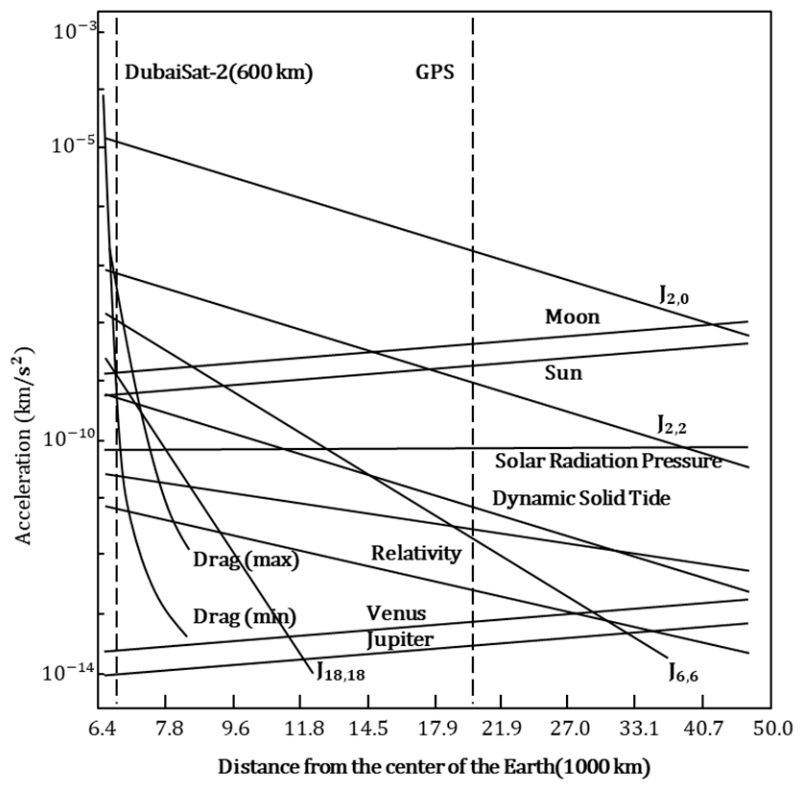

Figure 1. Order of magnitude of various perturbations of a satellite orbit. See text for further explanation [13].

This paper tries to improve the accuracy of the navigation solution by applying a more precise gravity model and the error characteristic of observation data to the orbit determination logic. The gravity model in the orbit model was switched from the EGM96 to the GGM03. The orbital frame of the orbit determination logic was converted from Earth-centered inertial (ECI) to radial, in-track, cross-track (RIC) to make the covariance of measurement represent the error characteristic of ionospheric time delay, which degrades the position accuracy of SPS up to $60 \mathrm{~m}$ [14]. This is the first 
application of GGM03 and RIC to the on-board real-time orbit determination logic. The efficacy of the RIC orbital frame and the GGM03 have been verified using the GPS simulator and the real GPS data of the CHAMP satellite, respectively. Improved on-board orbit determination logic has been applied to the GPS-12 in DubaiSat-2, and its performance is verified in this paper.

\section{Sensitivity of Orbital Frame for Orbit Determination}

An inertial frame of reference in classical physics and special relativity is a frame of reference in which a body with zero net force is acted upon; that is, such a body is at rest or is moving at a constant speed in a straight line [15]. In Principia, Newton published his three laws of motion, which are valid for a particle whose motion is observed in a reference frame that is fixed in space or is translating with a constant velocity. The inertial acceleration of Newton's third law is related to the inertial velocity and inertial position [16]. Gravitational and nongravitational force act on LEO satellites and can be integrated to calculate the position and velocity in the ECI frame. That is why most orbit determination logic including the orbit dynamics has been designed on the ECI frame. However, the ECI frame has a disadvantage in specifying the error characteristic of the measurement, such as ionospheric time delay. In this section, the characteristics of measurement error as well as the performance of on-board orbit determination logic with respect to each ECI and RIC frame are presented, respectively. The x-axis of the RIC frame, the radial axis, is directed from the unit vector of the satellite position; the y-axis, the in-track axis, is the direction of motion; and the z-axis, the cross-track axis, is directed from the unit vector of normal orbit, as shown in Figure 2.

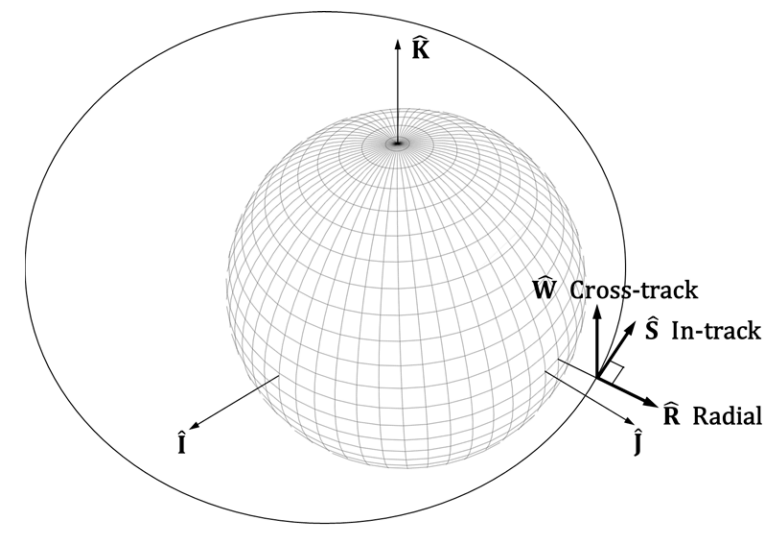

Figure 2. Conceptual diagram to show the definition of the radial, in-track, cross-track (RIC) frame.

\subsection{Test Configuration for Efficacy Verification: Sensitivity of Orbital Frame}

The test configuration using the GPS simulator (GNSS6700, Spirent Communications plc) is shown in Figure 3. The GPS simulator generates the motion of the LEO satellite by integrating acceleration, including gravitational force (Earth, Sun, and Moon) and nongravitational force (atmospheric drag, solar radiation pressure). At the same time, the simulator simulates the GPS signal and transmits a radio frequency $(\mathrm{RF})$ signal to the GPS receiver. The GPS receiver transfers the calculated navigation solution to the laptop. The performance of the navigation solution from the GPS receiver can be analyzed by comparison with the reference orbit (RO) that is generated in a GPS simulator. 


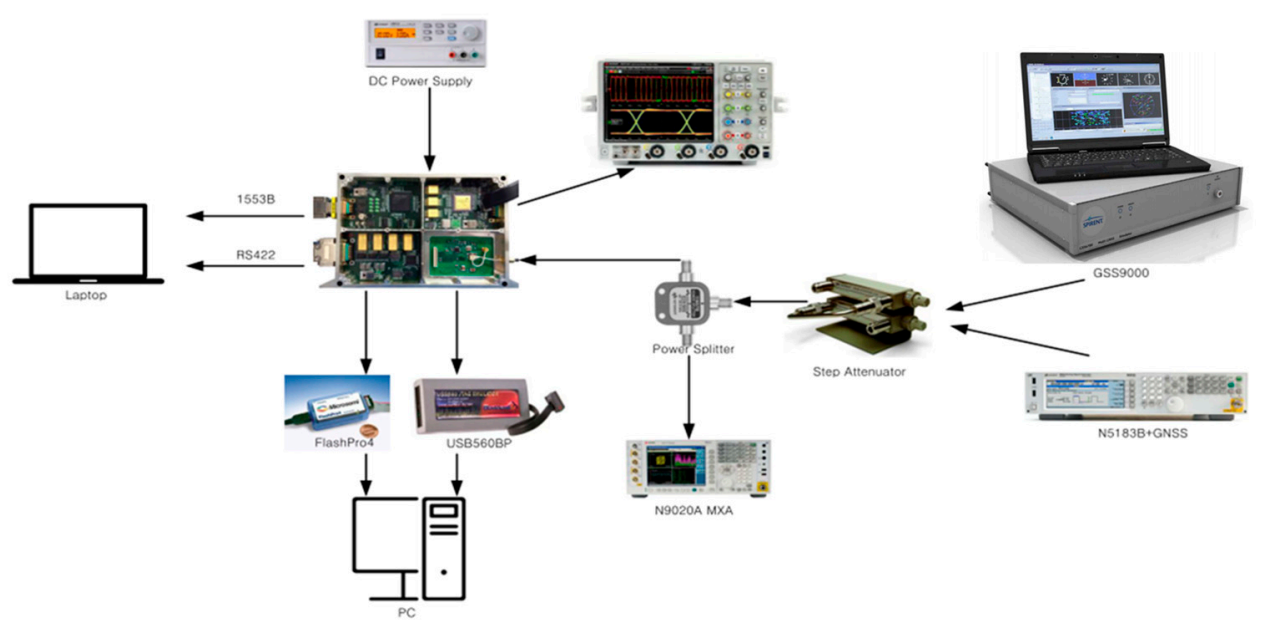

Figure 3. Test configuration using a GPS simulator to verify the performance of GPS-12.

\subsection{Efficacy of the RIC Frame for Identifying Ionospheric Time Delay}

Ionospheric time delay is still the largest source of error after selective availability (SA) is off [17]. The spaceborne GPS receiver, which is operated at an altitude of several hundred km or more, is very likely to receive navigation signals traveling through high electron-density regions, as can be seen in Figure 4a. That is because the antenna of the spaceborne GPS receiver will stare the high-electron-density region when the satellite does an attitude maneuver in the imaging or eclipse scenario. That is when the pseudo-range error due to the ionospheric time delay could be much bigger than that of the GPS receiver operated on the ground. The ionospheric time delay for the spaceborne GPS receiver at altitudes of $400 \mathrm{~km}, 500 \mathrm{~km}$, and $600 \mathrm{~km}$ can be estimated using the electron density from the International Reference Ionosphere (IRI), as shown in Figure $4 \mathrm{~b}$.

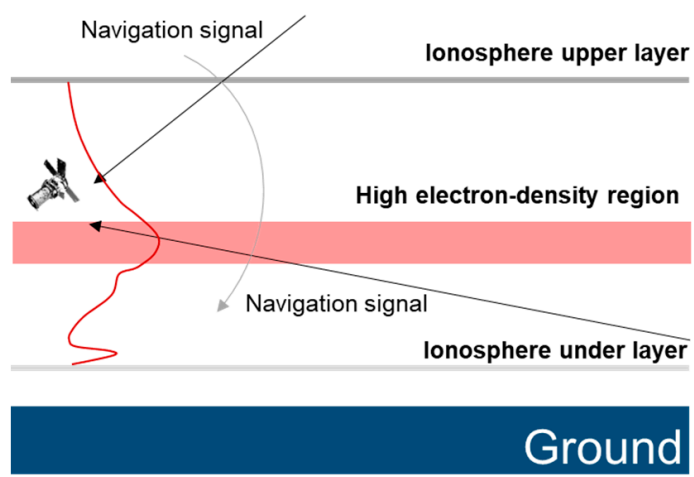

(a)

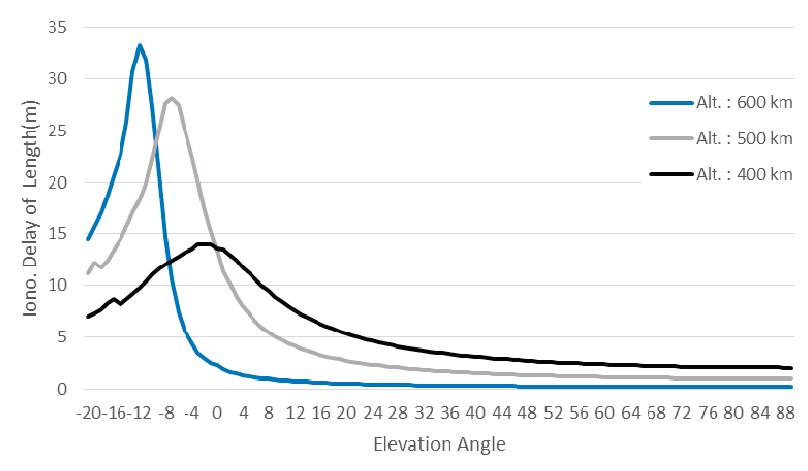

(b)

Figure 4. Features of ionospheric time delay affecting pseudo-range according to the incidence angle of the navigation signal [14]. (a) The practical tendency of ionospheric time delay can be summarized as being likely to receive the navigation signal from a negative incidence angle. (b) Peaks of time delays.

The ionospheric time delay from the low-elevation angle of the signal degrades the position accuracy up to several tens of meters [14]. When analyzing the error caused by ionospheric time delay with respect to the ECI frame, there is a disadvantage in that the error is distributed to each axis, as shown in Figure 5a. As a consequence, the covariance of measurement noise of the orbit determination logic should be on a big scale. However, the ionospheric time delay mainly affects the position vector, as shown in Figure 5b. Thus, if the orbit determination logic is designed on the orbital frame, which 
has a position vector like the RIC frame, the covariance of the measurement noise can be designed more precisely, and it will improve the performance of the navigation solutions.

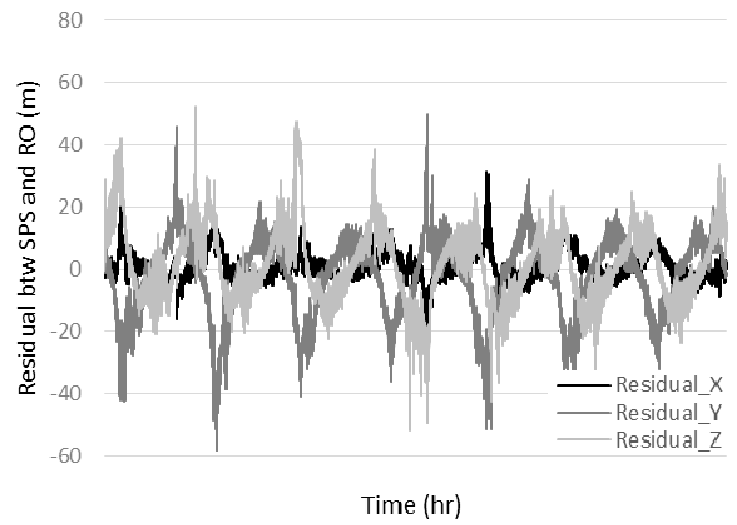

(a)

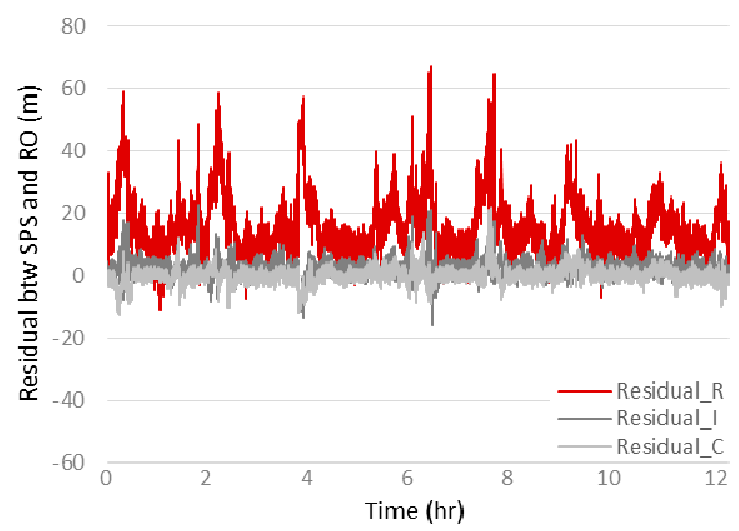

(b)

Figure 5. Ionospheric time delay represented according to the frame. If there are ionospheric time delay errors: (a) the error is distributed in all axes in the Earth-centered inertial (ECI) frame; (b) the error is focused in the radial axis of the RIC frame. The other axis appears to contain only noise error without the effect of ionospheric time delay.

\subsection{On-Board Orbit Determination Logic Design on RIC Orbital Frame}

This section suggests using orbit determination logic on the RIC orbital frame to improve the accuracy of the navigation solution while exploiting the HPOP orbit model. The state vector comprises the inertial position and velocity on ECI, simply:

$$
x_{E C I, k}=\left[\begin{array}{llllll}
P_{x} & P_{y} & P_{z} & V_{x} & V_{y} & V_{z}
\end{array}\right]_{E C I, k} .
$$

A flowchart for the extended Kalman filter approach is summarized in Figure 6. In the system dynamic model, $x_{k}$ is the state vector, $w_{k}$ is the process noise, $y_{k}$ is the observation data, and $v_{k}$ is the measurement noise. The observation data also comprises the inertial position and velocity, which is calculated by SPS logic using the pseudo-range and its rate. It is critical to prepare the coordinate transition matrix prior to the initiation of the orbit determination logic. The current coordinate transition matrix $\left(T_{R I C \rightarrow E C I, k}\right)$ can be calculated using the propagated states vector, and the previous coordinate transition matrix $\left(T_{R I C \rightarrow E C I, k-1}\right)$ can be calculated using the determined location. The covariance of measurement noise is designed to apply the feature of ionospheric time delay and is summarized in Table 1. As shown in Table 1, the covariance of the radial vector takes up the most ionospheric time delay, and the other axis is applied only to the noise component:

$$
R_{k}=\left[\begin{array}{cc}
\left(R_{r, k}\right)_{3 \times 3} & 0_{3 \times 3} \\
0_{3 \times 3} & \left(R_{v, k}\right)_{3 \times 3}
\end{array}\right]
$$




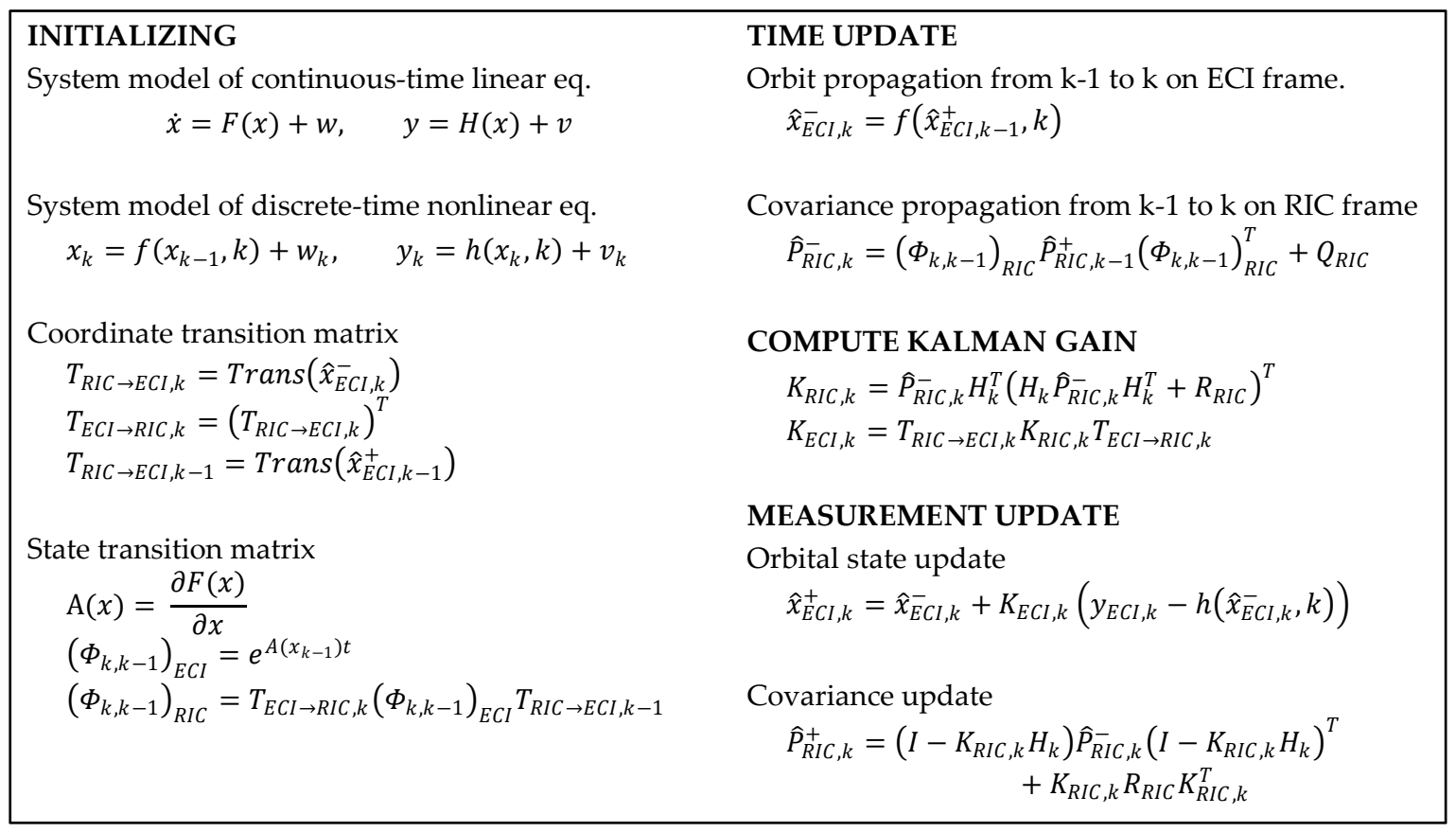

Figure 6. Orbit determination logic using extended Kalman filter on the RIC orbital frame.

Table 1. Design of covariance of measurement noise according to the orbital frame.

\begin{tabular}{|c|c|c|c|c|c|}
\hline \multicolumn{6}{|c|}{ Covariance of Measurement Noise $\left(\mathrm{km}^{2}\right)$} \\
\hline \multicolumn{3}{|c|}{ Frame: ECI } & \multicolumn{3}{|c|}{ Frame: RIC } \\
\hline Position: $R_{r, k}$ & & & Position: $R_{r, k}$ & & \\
\hline$\left[4.0 e^{-4}\right.$ & 0 & 0 & {$\left[5.0 e^{-4}\right.$} & 0 & 0 \\
\hline 0 & $4.0 e^{-4}$ & 0 & 0 & $2.5 e^{-5}$ & 0 \\
\hline$[0$ & 0 & $1.0 e^{-4}$ & 0 & 0 & $2.5 e^{-5}$ \\
\hline Velocity: $R_{v, k}$ & & & Velocity: $R_{v, k}$ & & \\
\hline$\left[2.0 e^{-8}\right.$ & 0 & 0 & {$\left[2.0 e^{-8}\right.$} & 0 & 0 \\
\hline 0 & $2.0 e^{-8}$ & 0 & 0 & $2.0 e^{-8}$ & 0 \\
\hline 0 & 0 & $2.0 e^{-8}$ & 0 & 0 & $2.0 e^{-8}$ \\
\hline
\end{tabular}

\subsection{Sensitivity Analysis Result: Orbital Frame}

This section describes the efficacy of the orbital frame sensitivity affecting on-board real-time orbit determination logic using the GPS simulator. To confirm its effectiveness in reducing the ionospheric time delay, the accuracy of the orbit determination logic was analyzed on the RIC frame using the reference orbit from the GPS simulator. Moreover, the performance of the orbit determination logic using the RIC orbital frame was compared with its accuracy using the ECI orbital frame, which was summarized in previous research [12]. The system model in Figure 6 was the orbit model used in previous research, composed of the gravity model (Earth gravity, EGM96 (up to the 40th degree and order), third body) and the non-gravity model (atmospheric drag, solar radiation pressure) [12]. The RIC orbital frame was applied to the on-board orbit determination logic of GPS-12 in DubaiSat-2, and its practical performance is verified in Section 4.

Table 2 and Figure 7 summarize the performance of orbit determination (OD) logic on both the ECI and RIC frames by comparison with the reference orbit (RO) that is generated in a GPS simulator. Observation data is also shown in Figure 7 to confirm the performance of the OD more clearly. The position accuracy improved from $18.07 \mathrm{~m}(1 \sigma)$ to $11.24 \mathrm{~m}(1 \sigma)$, about 37.5\%, after applying the RIC orbital frame. In addition, the peak improved from $38.59 \mathrm{~m}$ to $22.29 \mathrm{~m}$, about $42.23 \%$. In terms of the position accuracy of each RIC axis, the accuracy improved mainly in the radial and in-track axes. The accuracy of the in-track axis improved more than $40 \%$, from $9.30 \mathrm{~m}(1 \sigma)$ to $5.48 \mathrm{~m}(1$ 
$\sigma)$. The performance improvement of on-board orbit determination logic is due to the use of the precise covariance of measurement. The position accuracy improvement led to better velocity accuracy. The velocity improved from $25.51 \mathrm{~mm} / \mathrm{s}(1 \sigma)$ to $11.86 \mathrm{~mm} / \mathrm{s}(1 \sigma)$, about $53.5 \%$, and peak improved from $53.09 \mathrm{~mm} / \mathrm{s}$ to $38.12 \mathrm{~mm} / \mathrm{s}$. Similar to the position accuracy, the velocity accuracy improved greatly at the radial and in-track axes. In particular, the accuracy of the in-track axis improved from $10.53 \mathrm{~mm} / \mathrm{s}(1 \sigma)$ to $4.57 \mathrm{~mm} / \mathrm{s}(1 \sigma)$, about $57 \%$.

Table 2. Performance analysis results of on-board orbit determination with respect to reference orbit from GPS simulator.

\begin{tabular}{|c|c|c|c|c|c|c|c|c|c|}
\hline \multirow{5}{*}{ 营 } & \multirow[b]{3}{*}{ Avg. (m) } & \multicolumn{4}{|c|}{ Orbital Frame: ECI } & \multicolumn{4}{|c|}{ Orbital Frame: RIC } \\
\hline & & \multirow{2}{*}{$\begin{array}{c}\text { Radial } \\
-9.58\end{array}$} & \multirow{2}{*}{$\begin{array}{c}\text { In-Track } \\
5.09\end{array}$} & \multicolumn{2}{|c|}{ Cross-TrackRange } & \multirow{2}{*}{$\begin{array}{c}\text { Radial } \\
-6.28\end{array}$} & \multirow{2}{*}{$\begin{array}{c}\text { In-Track } \\
2.84\end{array}$} & \multicolumn{2}{|c|}{ Cross-TrackRange } \\
\hline & & & & -0.05 & 12.04 & & & -0.05 & 7.49 \\
\hline & Std. (m) & 6.26 & 4.21 & 2.57 & 6.03 & 4.26 & 2.64 & 2.61 & 3.75 \\
\hline & Peak (m) & -33.44 & 20.67 & -6.09 & 38.59 & -20.90 & 18.50 & -6.30 & 22.29 \\
\hline \multirow{3}{*}{$\begin{array}{l}\frac{D}{0} \\
\frac{0}{0} \\
>\end{array}$} & Avg. (mm/s) & -14.63 & 3.57 & -0.02 & 14.90 & -6.63 & 1.87 & -0.09 & 6.30 \\
\hline & Std. $(\mathrm{mm} / \mathrm{s})$ & 10.90 & 6.96 & 4.78 & 12.61 & 4.10 & 2.70 & 3.09 & 5.56 \\
\hline & Peak (mm/s) & -47.98 & 21.05 & 12.31 & 53.09 & -30.21 & 20.11 & 7.21 & 38.12 \\
\hline
\end{tabular}

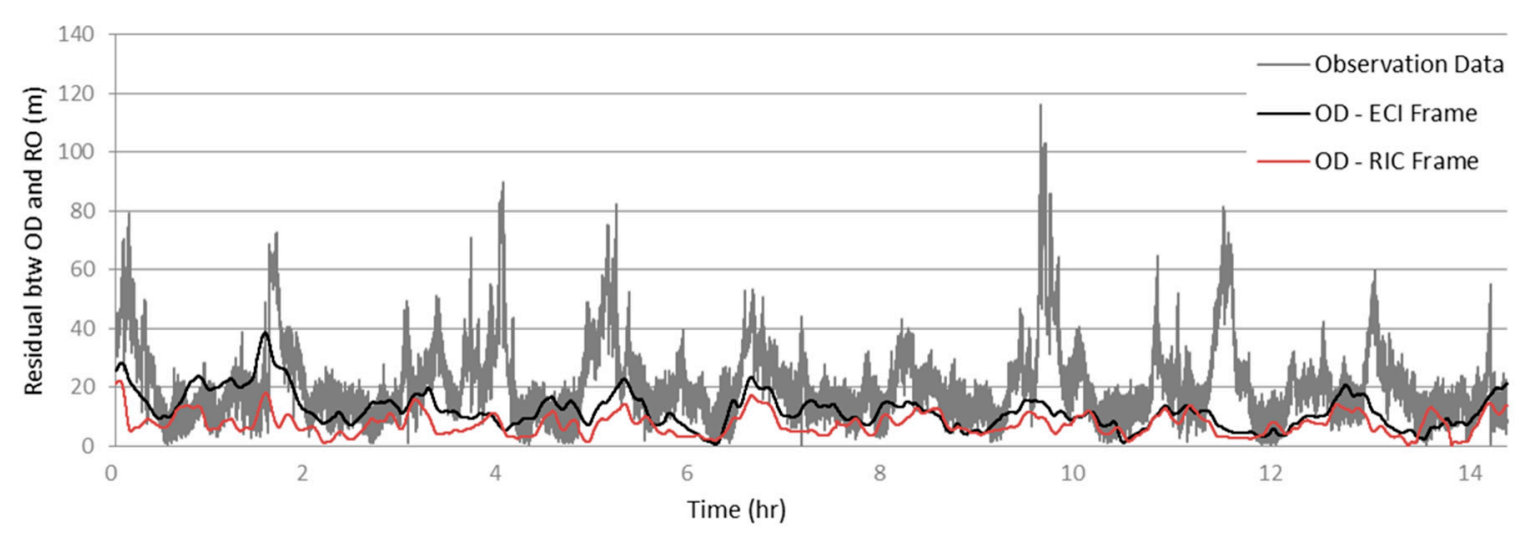

Figure 7. Superiority and inferiority of the orbital frame when orbit determination logic is performed. The accuracy of orbit determination logic improved after applying the RIC frame, and local maximum errors that are seriously affected by ionospheric time delay were also reduced.

\section{Sensitivity of Gravity Model for Orbit Determination}

As can be seen in Figure 1, the acceleration of gravity takes up most of the perturbation force that affects LEO satellites. The launch of Sputnik in 1957 marked the advent of satellite geodesy and offered an easier way to create a global gravity model of the Earth by tracking satellites and measuring perturbations in their orbits. In the early 1990s, GPS quickly advanced the field of satellite geodesy by offering continuous, accurate satellite tracking [18]. These technologies allowed the creation of much more accurate static gravity field models of the Earth. In 1998, EGM96 was released, the result of a joint collaboration between the NASA Goddard Space Flight Center (GSFC) and the National Imagery and Mapping Association (NIMA), using years of satellite tracking and terrestrial gravity measurements [19]. Figure 8 shows the history of changes in the gravity model. EGM96 had been the most accurate Earth gravity model until 2000. GGM03, which was based on the analysis of four years of GRACE in-flight data, was released in 2008. GGM03 is much more accurate than EGM96 when comparing the residual RMS of laser ranging [20]. In this section, GGM03 is applied to the on-board real-time orbit determination logic to improve its performance for the first time. Moreover, the sensitivity of the gravity model is analyzed according to EGM96 and GGM03. 


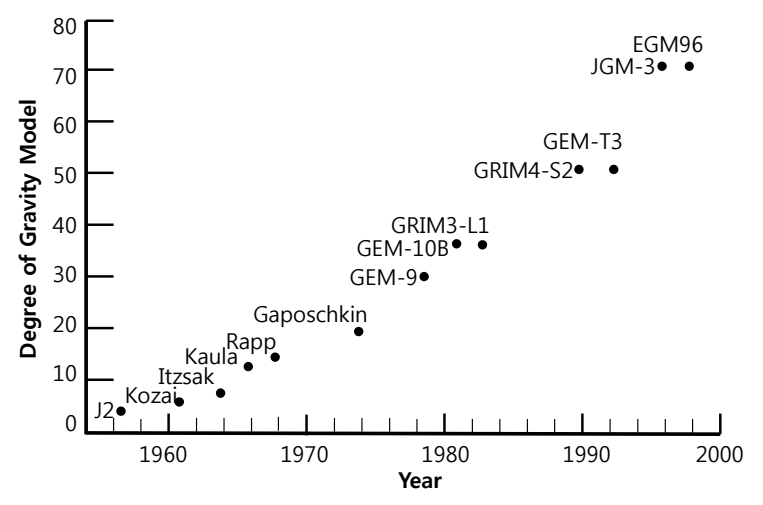

Figure 8. Evolution of complexity in Earth gravity models [13].

\subsection{Test Configuration for Efficacy Verification: Sensitivity of Gravity Model}

The performance of the on-board orbit determination logic was verified using real GPS data from the CHAMP satellite, as shown in Figure 9. The results of the orbit determination logic were compared with the precise orbit ephemeris (POE) of the CHAMP satellite. The position and velocity calculated by SPS logic using pseudo-range and its rate were used as the observation data, as usual.

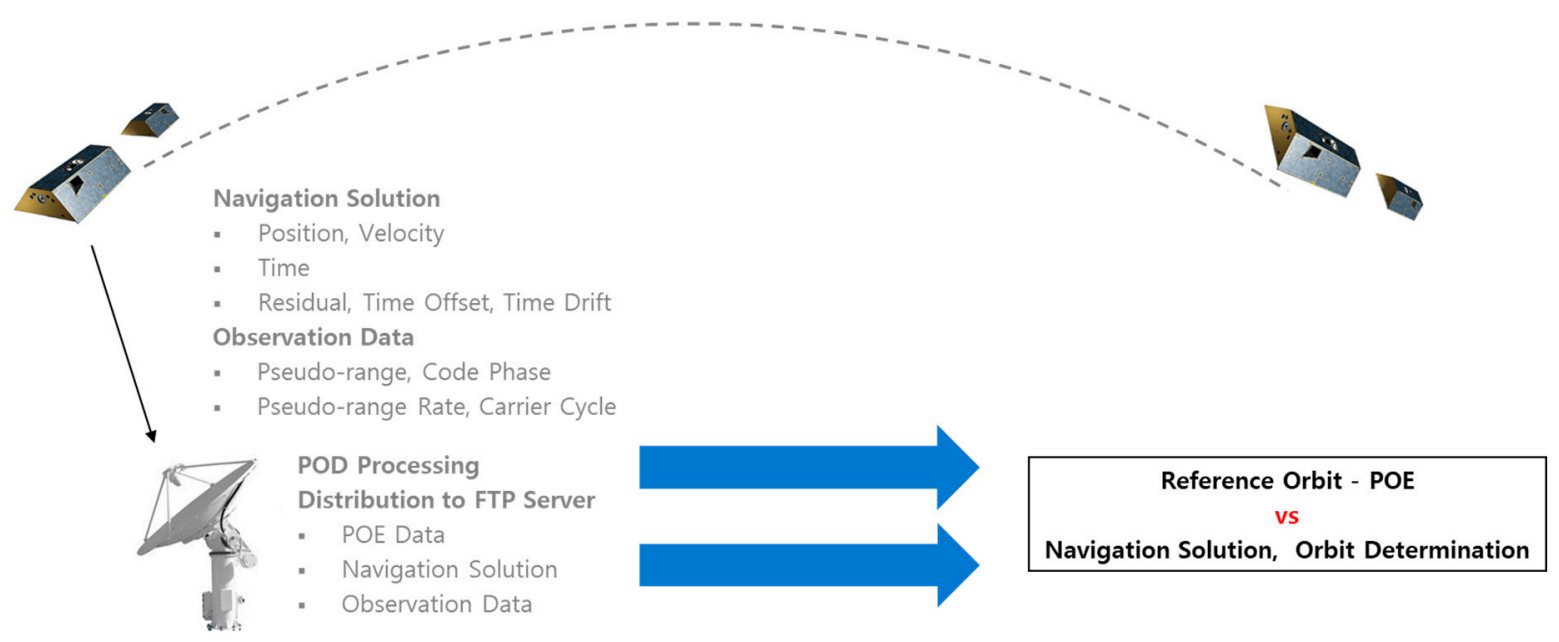

Figure 9. Test configuration using real GPS data from the Challenging Minisatellite Payload (CHAMP) satellite to verify the performance of the orbit determination logic. POE-precise orbit ephemeris.

\subsection{Efficacy of Gravity Model for Orbit Propagation}

This section describes the accuracy of the orbit propagation (OP) model. As mentioned before, GGM is much more accurate than EGM96 [21]. To analyze the accuracy when the orbit model is applied to the LEO satellite, not the geoid, the propagated orbit was fitted to the POE of CHAMP for a day (25 November 2007) by using differential correction and the least squares method. The state of the least squares method includes the position, velocity, coefficient of atmospheric drag (Cd), and solar radiation $(\mathrm{Cr})$. The least squares method with differential correction was summarized in previous research [22], and the analysis results are summarized in Table 3 and Figure 10 using the Systems Tool Kit (STK). Table 3 shows the fitted accuracy at 1 sigma for the GGM03, EGM96, Joint Gravity Model (JGM)2, JGM3, World Geodetic System (WGS)84, and WGS84-EGM96 orbit models. Figure 10 represents just GGM03, EGM96, JGM3, and WGS84. The degree and order of Earth gravity are 70 and 70, respectively. The orbit model includes atmospheric drag (Harris-Priester density model), third body (Sun and Moon), and solar radiation pressure (dual cone). The fitting results show that GGM03 is much more accurate than the other gravity models. The position accuracy of GGM03 is about $1.6 \mathrm{~m}$. 
and the velocity accuracy is about $0.4 \mathrm{~mm} / \mathrm{s}$. Compared to the least accurate orbit model, WGS84 is about 16 times worse than GGM03.

Table 3. Performance analysis results in 1 sigma of various orbit propagation models with respect to the reference orbit, CHAMP precise orbit ephemeris (POE).

\begin{tabular}{ccccccc}
\hline & GGM03 & EGM96 & JGM2 & JGM3 & WGS84 & WGS84-EGM96 \\
\hline 3D Position (m) & 1.61 & 13.48 & 16.43 & 15.28 & 27.11 & 10.82 \\
\hline 3D Velocity (mm/s) & 0.42 & 3.25 & 3.58 & 3.23 & 9.72 & 3.31 \\
\hline
\end{tabular}

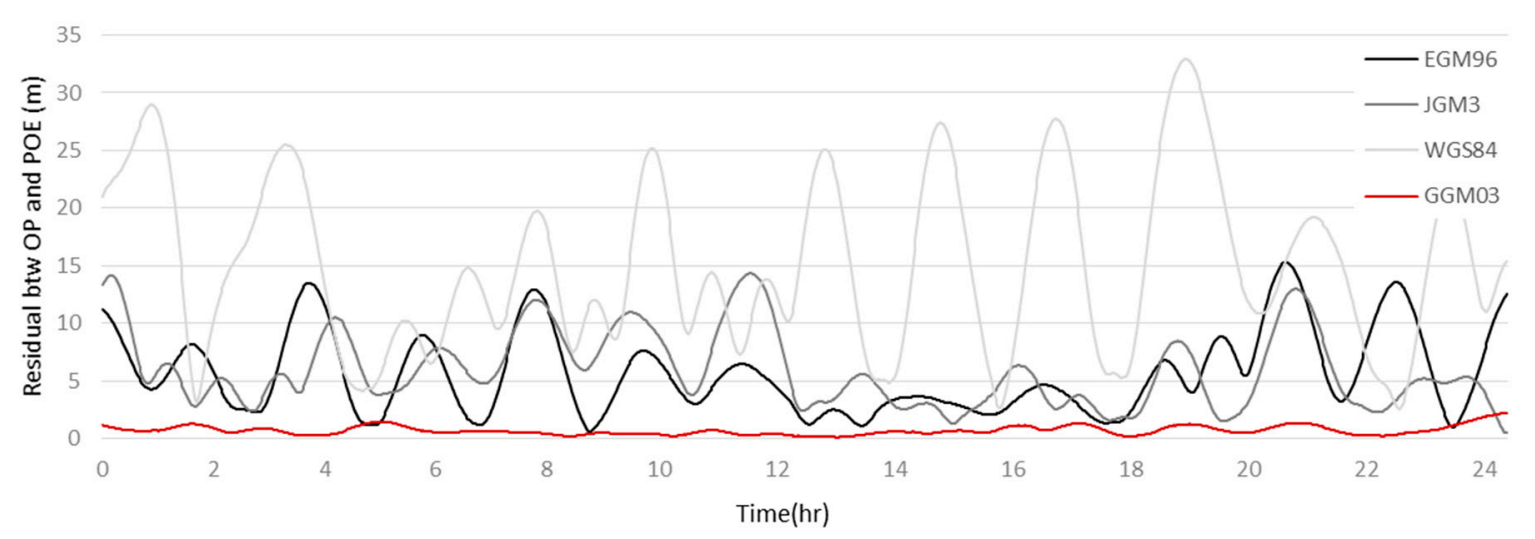

Figure 10. Performance analysis results of various orbit propagation models with respect to the reference orbit, CHAMP POE.

\subsection{Sensitivity Analysis Result: Gravity Model}

This section describes the efficacy of the gravity model's sensitivity affecting the orbit determination logic using the real GPS data from the CHAMP satellite from 25 November 2007. To confirm its effectiveness, the accuracy of the orbit determination logic was analyzed on the RIC frame using the reference orbit from the CHAMP POE. In addition, the performance improvement of the orbit determination logic using GGM03 was compared with the accuracy using EGM96. The system model shown in Figure 6 is exactly the same as the orbit model described in Section 2, but for the gravity model. The gravity model was switched from EGM96 to GGM03. GGM03 was applied to the on-board orbit determination logic of GPS-12 in DubaiSat-2, and its practical performance is analyzed in Section 4.

Table 4 and Figure 11 summarize the performance of the orbit determination logic on EGM96 and GGM03 by comparison with the reference orbit from the CHAMP POE. The position accuracy using SPS logic is about $5.7 \mathrm{~m}(1 \sigma)$. The orbit determination logic with GGM03 improved the position accuracy about $62 \%$, from $5.7(1 \sigma) \mathrm{m}$ to $2.4 \mathrm{~m}(1 \sigma)$, while EGM96 improved the position accuracy only about $35 \%$, from $5.7 \mathrm{~m}(1 \mathrm{\sigma})$ to $3.8 \mathrm{~m}(1 \mathrm{\sigma})$. The peak position improved about $80 \%$, from $27.19 \mathrm{~m}$ to $5.7 \mathrm{~m}$, after applying GGM03, while the peak only improved about $52 \%$, from $27.19 \mathrm{~m}$ to $13.05 \mathrm{~m}$, using EGM96. In addition, the velocity accuracy of the orbit determination logic improved about $76 \%$, from $4.6 \mathrm{~mm} / \mathrm{s}(1 \sigma)$ to $1.1 \mathrm{~mm} / \mathrm{s}(1 \mathrm{\sigma})$, after applying GGM03. The peak of velocity improved about $85 \%$, from $15.78 \mathrm{~mm} / \mathrm{s}$ to $2.36 \mathrm{~mm} / \mathrm{s}$. The improved velocity ratio is better than the position. The axial features on the RIC frame in the accuracy analysis results are the same as those in Section 2. Table 4 shows that the accuracy of the in-track position improved the most, from $2.5 \mathrm{~m}(1 \sigma)$ to $0.5 \mathrm{~m}(1 \sigma)$ and $2.9 \mathrm{~mm} / \mathrm{s}(1 \sigma)$ to $0.4 \mathrm{~mm} / \mathrm{s}(1 \sigma)$. The accuracy of fitting results in Section 3.2 seems to be better than in this section, but that is because Section 3.2 used the CHAMP POE. 
Table 4. Performance analysis results of on-board orbit determination with respect to the reference orbit from CHAMP POE.

\begin{tabular}{|c|c|c|c|c|c|c|c|c|c|}
\hline \multirow{5}{*}{ 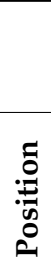 } & & \multicolumn{4}{|c|}{ Gravity Model: EGM96 } & \multicolumn{4}{|c|}{ Gravity Model: GGM03 } \\
\hline & & \multirow{2}{*}{$\begin{array}{c}\text { Radial } \\
-1.66\end{array}$} & \multirow{2}{*}{$\begin{array}{c}\text { In-Track } \\
1.11\end{array}$} & \multicolumn{2}{|c|}{ Cross-TrackRange } & \multirow{2}{*}{$\begin{array}{c}\text { Radial } \\
-1.31\end{array}$} & \multirow{2}{*}{$\begin{array}{c}\text { In-Track } \\
-0.02\end{array}$} & \multicolumn{2}{|c|}{ Cross-TrackRange } \\
\hline & Avg. (m) & & & 0.17 & 2.48 & & & 0.06 & 1.69 \\
\hline & Std. (m) & 1.33 & 1.36 & 0.74 & 1.40 & 0.97 & 0.5 & 0.70 & 0.76 \\
\hline & Peak (m) & 1.96 & 7.36 & 4.14 & 13.05 & 1.26 & 1.26 & 1.93 & 5.66 \\
\hline \multirow{3}{*}{ 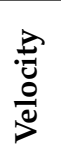 } & Avg. $(\mathrm{mm} / \mathrm{s})$ & -1.31 & 1.43 & -0.23 & 2.19 & 0.07 & -0.03 & -0.26 & 0.32 \\
\hline & Std. $(\mathrm{mm} / \mathrm{s})$ & 2.93 & 1.45 & 1.05 & 2.41 & 1.01 & 0.39 & 0.93 & 0.78 \\
\hline & Peak (mm/s) & 14.61 & 5.72 & 3.21 & 15.78 & 3.08 & 1.06 & 1.82 & 2.36 \\
\hline
\end{tabular}

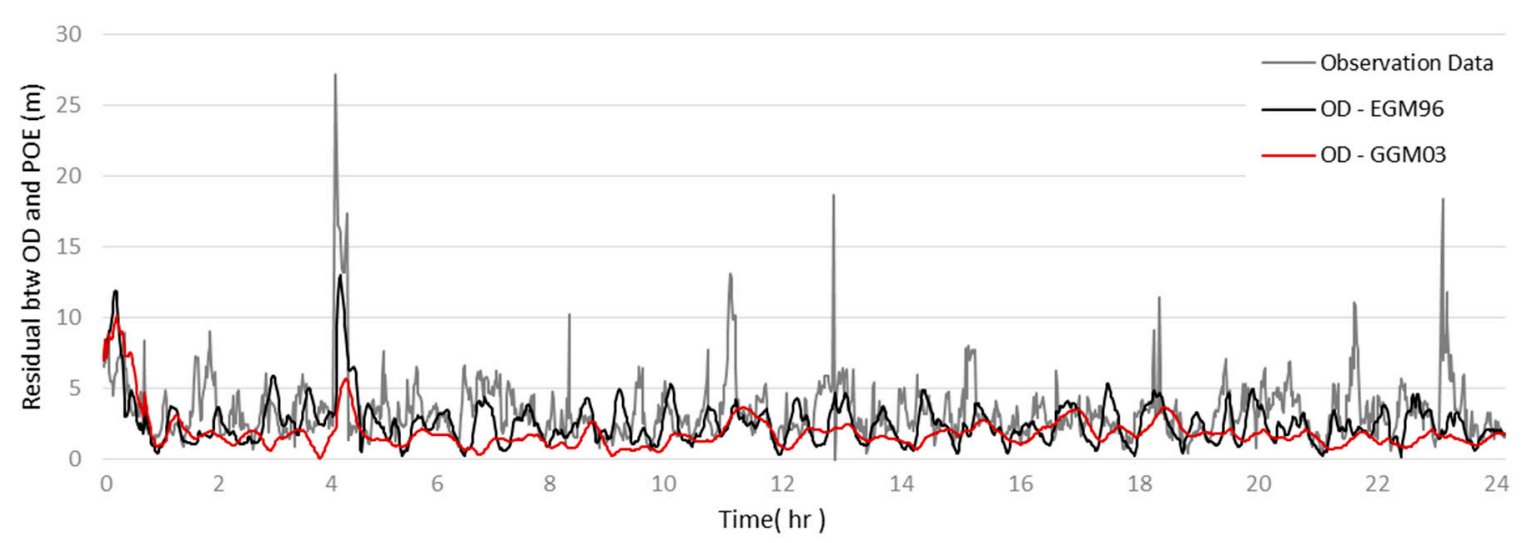

Figure 11. Superiority and inferiority of the gravity model when performing orbit determination logic. The accuracy of orbit determination logic improved after applying GGM03, and local maximum errors, which are seriously affected by the ionospheric time delay, were also reduced successfully.

\section{In-orbit Performance of On-board Real-time Orbit Determination Logic}

This paper suggests that both the RIC orbital frame and the precise gravity model (GGM03) improve the on-board real-time orbit determination logic. The efficacy of both methods was also verified using the GPS simulator and the real GPS data of the CHAMP satellite, in Sections 2 and 3. The Satrec Initiative has been trying to apply both methods to the GPS-12 on the DubaiSat-2 satellite. DubaiSat-2 was launched on 21 November 2013. Its mission orbit was designed as a Sun-synchronous orbit at an altitude of $600 \mathrm{~km}$ and a local time of descending node of 10:30. The total mass of the satellite is less than $300 \mathrm{~kg}$.

Both methods were applied to GPS-12 on DubaiSat-2 after 10 November 2018, and the performance of the on-board orbit determination logic was analyzed. Table 5 summarizes and describes the orbit models applied to the orbit determination logic of GPS-12. The determined navigation solution using orbit determination logic can be acquired from the satellite when the ground station contacts the satellite. Then, the reference orbit can be acquired by post-processing the navigation solution using the Orbit Determination Tool Kit (ODTK). The residual of the determined navigation solution can be calculated using the reference orbit, as shown in Figure 12. 
Table 5. Summary of orbit model including gravity and non-gravity models. The orbit model was operated practically in the on-board real-time orbit determination logic of GPS-12.

\begin{tabular}{|c|c|c|}
\hline Orbit Item & Subitem & Orbit Model of Orbit Determination Logic \\
\hline \multirow{3}{*}{ Gravity } & Model & GGM03 \\
\hline & Degree & 40 \\
\hline & Order & 40 \\
\hline \multirow{4}{*}{ Atmospheric drag } & $\mathrm{Cd}$ & 1.2 \\
\hline & Area/mass ratio $\left(\mathrm{m}^{2} / \mathrm{kg}\right)$ & 0.006 \\
\hline & Atm. density model & Harris-Priester density model \\
\hline & Satellite mass $(\mathrm{kg})$ & 300 \\
\hline \multirow{3}{*}{ Solar radiation pressure } & $\mathrm{Cr}$ & 0.705 \\
\hline & Area/mass ratio $\left(\mathrm{m}^{2} / \mathrm{kg}\right)$ & 0.006 \\
\hline & Shadow model & Dual cone \\
\hline \multirow{2}{*}{ Third body gravity } & Sun & Low-Precision Solar Coordinate [13] \\
\hline & Moon & Low-Precision Lunar Coordinate [13] \\
\hline
\end{tabular}

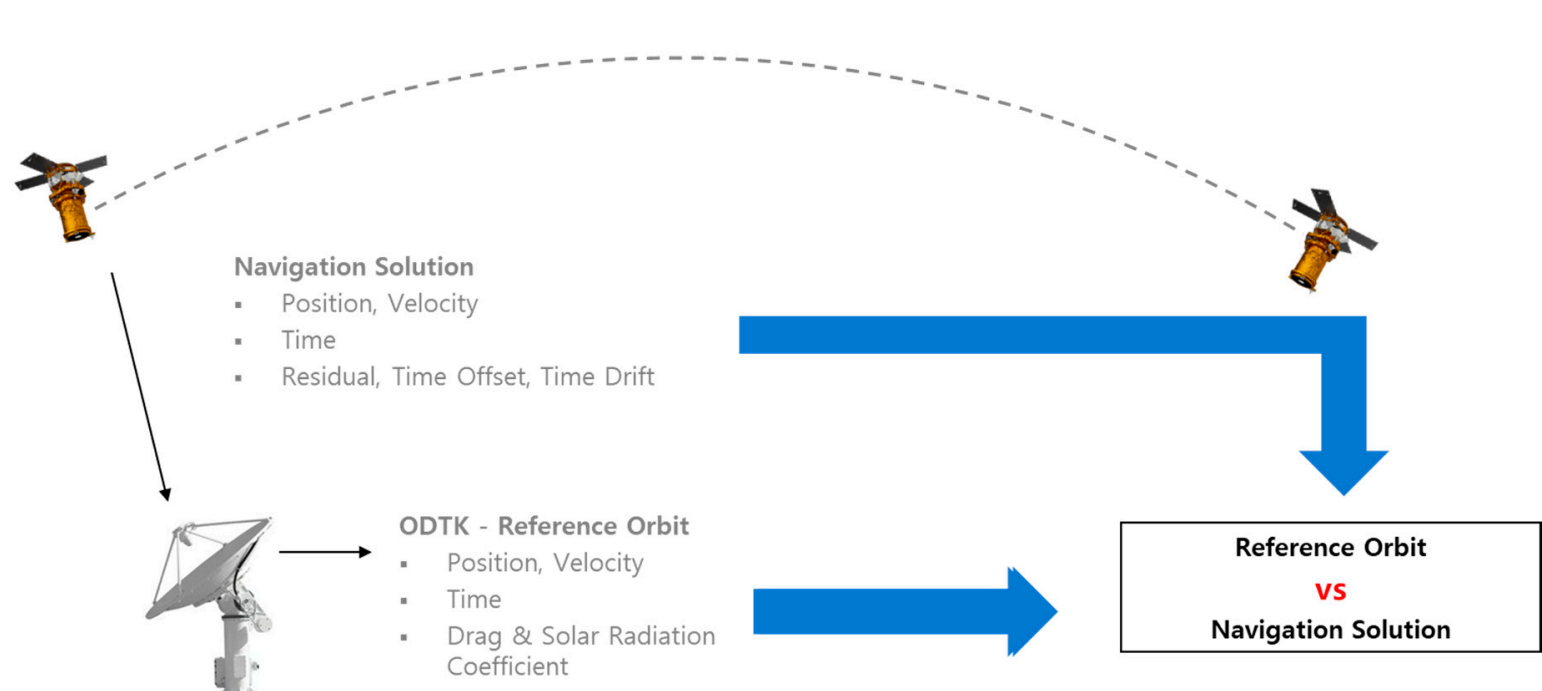

Figure 12. Test configuration using the navigation solution from the DubaiSat- 2 satellite to verify the performance of the on-board real-time orbit determination logic of GPS-12. ODTK-Orbit Determination Tool Kit.

The ODTK has high reliability and is used widely in many fields [23]. The ODTK can generate the reference orbit by using the navigation solution of the GPS receiver together with the coefficient of atmospheric drag $(\mathrm{Cd})$ and solar radiation pressure $(\mathrm{Cr})$. The analysis results before and after applying both methods are summarized in Table 6 and Figure 13. Figure 13 also contains the orbit determination result using the simple J2 gravity model to compare how much the orbit determination logic improves the navigation solution. As can be seen in Figure 13, the orbit determination logic with EGM96 and the ECI orbital frame improved the accuracy. To confirm its effectiveness, the accuracy of the orbit determination logic was analyzed on the RIC frame using the reference orbit. The analysis results show that the average, standard deviation, and peak improved for both position and velocity. The position improved about $59 \%$, from $12.6 \mathrm{~m}(1 \mathrm{\sigma})$ to $5.1 \mathrm{~m}(1 \sigma)$, and the velocity improved about $57 \%$, from $13.7 \mathrm{~mm} / \mathrm{s}(1 \sigma)$ to $5.9 \mathrm{~mm} / \mathrm{s}(1 \sigma)$. The improvement ratio is reasonable, considering the results in Sections 2 and 3. The position and velocity accuracy for each axis appear to be similar, which means that the ionospheric time delay was removed efficiently. Figure 13 shows that there are 28 local 
maxima. These local maxima could have occurred by the ionospheric time delay, because the delay is the biggest error source and should be maximized when the incidence angle of the navigation signal is less than $0^{\circ}$, as shown in Figure 4 . Attitude maneuvers such as the eclipse scenario of satellites make the spaceborne GPS receiver have navigation signals from a negative incidence angle. The satellite does the eclipse scenario twice during an orbit period, and DubaiSat-2 orbits around Earth about fourteen times a day. So there is likely to be 28 local maxima. Some position error by the ionospheric time delay remains when applying GGM03 and the RIC orbital frame, but the position accuracy greatly improves. However, some local maxima still remain.

Table 6. Performance analysis results of on-board real-time orbit determination logic of the GPS-12 on DubaiSat-2 with respect to the reference orbit using Orbit Determination Tool Kit (ODTK).

\begin{tabular}{|c|c|c|c|c|c|c|c|c|c|}
\hline & & \multicolumn{4}{|c|}{ Orbital Frame: ECI; Gravity Model: EGM96 } & \multicolumn{4}{|c|}{ Orbital Frame: RIC; Gravity Model: GGM03 } \\
\hline & & Radial & In-Track & Cross-Track & Range & Radial & In-Track & Cross-Track & Range \\
\hline \multirow{3}{*}{ : } & Avg. (m) & -0.016 & -0.17 & -0.012 & 8.61 & 0.003 & 0.01 & 0.25 & 3.44 \\
\hline & Std. (m) & 5.71 & 5.81 & 4.89 & 4.04 & 2.39 & 1.99 & 2.25 & 1.74 \\
\hline & Peak (m) & 16.94 & 21.86 & 14.58 & 26.38 & 8.44 & 6.02 & 6.09 & 11.57 \\
\hline \multirow{3}{*}{$\frac{D}{\stackrel{D}{0}}$} & Avg. (mm/s) & 0.17 & -0.12 & 0.41 & 8.49 & 0.015 & -0.09 & -1.14 & 3.85 \\
\hline & Std. $(\mathrm{mm} / \mathrm{s})$ & 5.37 & 5.74 & 6.11 & 5.22 & 2.42 & 2.01 & 2.83 & 2.08 \\
\hline & Peak (mm/s) & 19.21 & 28.53 & 19.51 & 32.51 & 8.22 & 5.43 & 7.94 & 16.08 \\
\hline
\end{tabular}

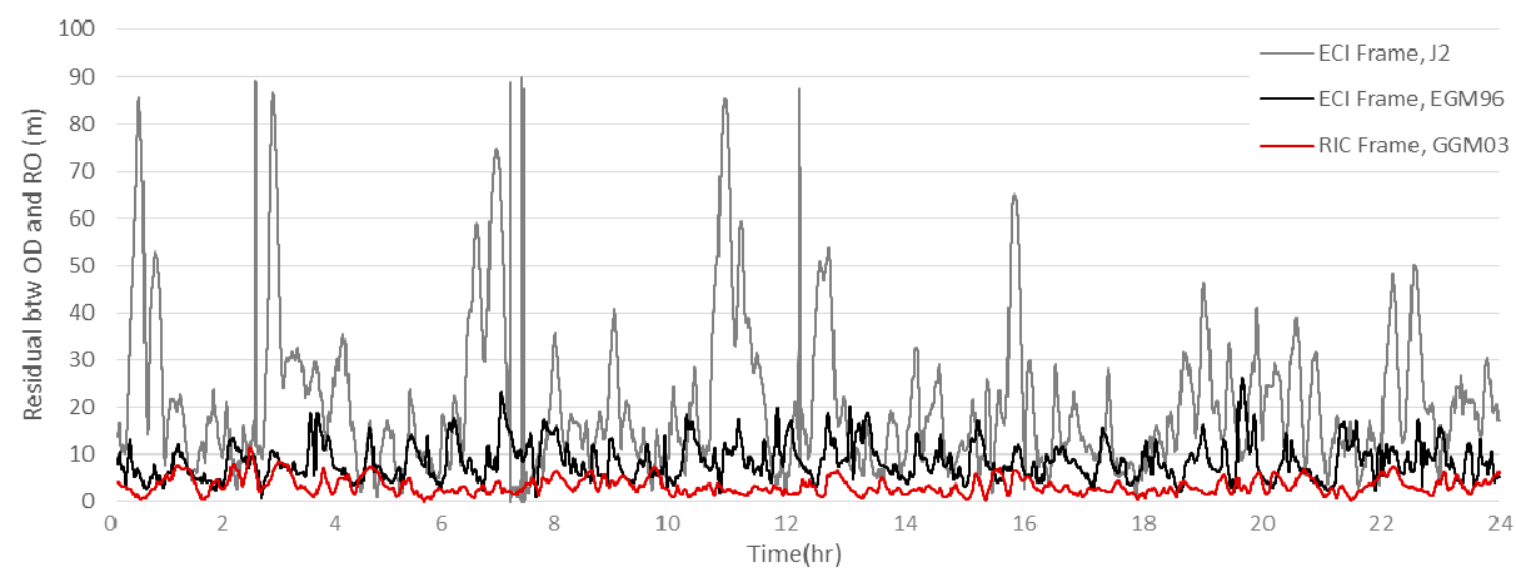

Figure 13. Advantages of applying the RIC orbital frame and GGM03 to the on-board real-time orbit determination logic of GPS-12 in DubaiSat-2. The accuracy of the determined position and velocity are improved. The peak, which might be affected by the ionospheric time delay, was also reduced successfully.

\section{Conclusions}

This paper focuses on improving the position and velocity accuracy of navigation solutions by suggesting two methods of switching the orbital frame from ECI to RIC and improving the gravity model from EGM96 to GGM03. The RIC orbital frame makes the covariance of measurement represent the error characteristic of ionospheric time delay well, and GGM03 upgrades the accuracy of orbit dynamics. The efficacy of the RIC orbital frame and the GGM03 have been verified using the GPS simulator and the real GPS data of the CHAMP satellite, respectively. After applying the RIC orbital frame to the on-board orbit determination logic, the position accuracy improved about $37.5 \%$, and the velocity improved about $53.5 \%$. In addition, after upgrading the gravity model to GGM03, the position accuracy improved by about $37 \%$, and the velocity accuracy improved by about $76 \%$. Both of these methods were applied to the orbit determination logic of GPS-12 in DubaiSat-2, and then the results of the determined navigation solution were analyzed. The results show that the on-board real-time 
orbit determination logic using the RIC frame and GGM03 improved the accuracy of the position by about $59 \%$, from $12.6 \mathrm{~m}(1 \sigma)$ to $5.1 \mathrm{~m}(1 \sigma)$, and improved the velocity by about $57 \%$, from $13.7 \mathrm{~mm} / \mathrm{s}$ $(1 \sigma)$ to $5.9 \mathrm{~mm} / \mathrm{s}(1 \sigma)$. It is expected that the improved position and velocity will be helpful in the progress of orbit propagation. Accurate orbit propagation is critical to missions such as SAR imaging and satellite automation. However, the peaks of position and velocity were beyond $10 \mathrm{~m}$ and $1 \mathrm{~cm} / \mathrm{s}$, respectively. To improve the peak performance, the on-board orbit determination logic needs to be upgraded further.

Author Contributions: All authors conceived the study. S.H. analyzed the orbit solutions via observation data from the CHAMP satellite. A.M.A.S. is in charge of DubaiSat-2 operation. Developing on-board real-time orbit determination logic, as well as the performance validation of the navigation solution from DubaiSat-2 were performed by E.K.

Funding: This research received no external funding.

Acknowledgments: We are grateful to the anonymous reviewers, whose insightful comments have notably improved the clarity of the presentation of the paper. We are greatly appreciative to the MBCRS for providing logs which downloaded from DubaiSat-2.

Conflicts of Interest: The authors declare no conflict of interest.

\section{References}

1. Birmingham, W.P.; Miller, B.L.; Stein, W.L. Experimental Results of Using the GPS for Landsat 4 Onboard Navigation. J. Inst. Navig. 1983, 30, 244-251. [CrossRef]

2. Heuberger, H.; Church, L. Landsat/4/Global Positioning System navigation results. Adv. Astronaut. Sci. 1984, 54, 589-602.

3. Fang, B.; Seifert, E. An evaluation of Global Positioning System data for Landsat-4 orbit determination. In Proceedings of the 23th Aerospace Sciences Meeting, Reno, NV, USA, 14-17 January 1985; p. 268.

4. Montenbruck, O.; Günther, C.; Graf, S.; Garcia-Fernandez, M.; Furthner, J.; Kuhlen, H. GIOVE-A initial signal analysis. GPS Solut. 2006, 10, 146-153. [CrossRef]

5. Misra, P.; Enge, P. Global Positioning System-Signals, Measurements, and Performance, 2nd ed.; Ganga Jammuna Press: Lincoln, MA, USA, 2006.

6. Yoon, J.C.; Lee, B.S.; Choi, K.H. Spacecraft orbit determination using GPS navigation solutions. Aerosp. Sci. Technol. 2000, 4, 215-221. [CrossRef]

7. Choi, E.J.; Yoon, J.C.; Lee, B.S.; Park, S.Y.; Choi, K.H. Development of spaceborne GPS receiver with real-time orbit determination using unscented kalman filter. In Proceedings of the IAC 2009, Dajeon, Korea, 12 October 2009.

8. Chiaradia, A.P.M.; Gill, E.; Montenbruck, O.; Kuga, H.K.; Prado, A.F.B.A. Algorithms for on-Board Orbit Determination Using GPS OBODE-GPS. DLR German Space Operations Center Oberpfaffenhofen; ResearchGate: Berlin, Germany, 2000.

9. Gill, E.; Montenbruck, O. On-Board Navigation System for the BIRD Satellite; Deutsches Zentrum fuer Luftund Raumfahrt: Berlin, Germany, 2002.

10. Montenbruck, O.; Swatschina, P.; Markgraf, M.; Santandrea, S.; Naudet, J.; Tilmans, E. Precision spacecraft navigation using a low-cost GPS receiver. GPS Solut. 2012, 16, 519-529. [CrossRef]

11. Kim, E.H.; Koh, D.W.; Chung, Y.S.; Park, S.B.; Jin, H.P.; Lee, H.W. Real Time On-board Orbit Determination Performance Analysis of Low Earth Orbit Satellites. J. Korean Soc. Aeronaut. Space Sci. 2015, 43, 10-20.

12. Kim, E.H.; Lee, B.H.; Park, S.B.; Jin, H.P.; Lee, H.W.; Jeong, Y.H. Performance Improvement of Real Time On-board Orbit Determination using High Precision Orbit Propagator. J. Korean Soc. Aeronaut. Space Sci. 2016, 44, 545-551.

13. Montenbruck, O.; Gill, E. Satellite Orbits, 1st ed.; Springer: Berlin, Germany, 2000.

14. Kim, E.H.; Koh, D.W.; AI Sayegh, A.M.; AI Midfa, I.A. Ionospheric Model Correction for Spaceborne GNSS Receiver. In Proceedings of the European Navigation Conference 2017, Lausanne, Switzerland, 9-12 May 2017; pp. 224-231.

15. Inertial Frame of Reference. Available online: https://en.wikipedia.org/wiki/Inertial_frame_of_reference (accessed on 25 June 2019). 
16. Wie, B. Space Vehicle Dynamics and Control, 2nd ed.; AIAA: Reston, VA, USA, 2008.

17. Tsui, J.B.Y. Fundamentals of Global Positioning System Receivers, 1st ed.; John Wiley \& Sons, Inc.: Hoboken, NJ, USA, 2000; Volume 173, pp. 102-105.

18. Nerem, R.S.; Jekeli, C.; Kaula, W.M. Gravity field determination and characteristics: Retrospective and prospective. J. Geophys. Res. 1995, 100, 15053-15074. [CrossRef]

19. Lemoine, F.G.; Kenyon, S.C.; Factor, J.K.; Trimmer, R.G.; Pavlis, N.K.; Chinn, D.S.; Cox, C.M.; Klosko, S.M.; Luthcke, S.B.; Torrence, M.H.; et al. The Development of the Joint NASA GSFC and NIMA Geopotential Model EGM96; NASA Goddard Space Flight Center: Greenbelt, MD, USA, 1998.

20. Cheng, M.; Ries, J.C.; Chambers, D.P. Evaluation of the EGM2008 Gravity Model. Newton's Bull. 2009, $4,18-25$.

21. Tapley, B.D.; Bettadpur, S.; Watkins, M.M.; Reigber, C. GRACE Gravity Model GGM01. Available online: http://www2.csr.utexas.edu/grace/gravity/ggm01/GGM01_Notes.pdf (accessed on 25 June 2019).

22. Kim, E.H. TLE Orbit Determination by using selectively applying GPS Navigation Solution. In Proceedings of the Korean Society for Aeronautical \& Space Sciences, Heraklion, Greece, 22-27 June 2014; pp. 623-627.

23. Vallado, D.A.; Hujsak, R.S.; Johnson, T.M.; Seago, J.H.; Woodburn, J.W. Orbit Determination using ODTK Version 6. In Proceedings of the European Space Astronomy Center 2010, Madrid, Spain, 3-6 May 2010.

(C) 2019 by the authors. Licensee MDPI, Basel, Switzerland. This article is an open access article distributed under the terms and conditions of the Creative Commons Attribution (CC BY) license (http://creativecommons.org/licenses/by/4.0/). 